\title{
Zwangssterilisierung im Nationalsozialismus
}

Zu dem Buch von Hans Georg Güse und Norbert Schmacke, Zwangssterilisiert. Verleugnet - vergessen. Zur Geschichte der nationalsozialistischen Rassenhygiene am Beispiel Bremens*

"Aussonderung sozialer Ballast-Existenzen «, »eugenische Aufartung der deutschen Volks-Substanz «, "Sterbehilfe für lebensunwertes Leben« - wer spricht diese Sprache? Wer hat die Begriffe geprägt? Nationalsozialistische Eugeniker oder Bevölkerungspolitiker und Gen-Technologen unserer Zeit? Beschäftigt man sich heute mit der Literatur zur NS-Rassenhygiene, so kommt einem manches bekannt vor: Wieder aktuell? Oder noch immer?

Betrachtet man die historischen Fotos, mit denen die Nationalsozialisten ihren Krieg gegen die Irrenpflege propagandistisch eingeleitet haben, die Bilder jener Opfer - taub, verkrüppelt, verzerrt -, dann fragt man sich, was in uns hängen geblieben ist von dieser Angst vor den sogenannten "Defekt-Menschen«. Wer von uns wagt es, einem Krüppel in die Augen zu schauen, ihn zu berühren? Wer hat jemals eine Aufbewahrungsanstalt für Schwerstbehinderte von innen gesehen? Einem Rollstuhlfahrer die Türe aufzuhalten, erzeugt schon Herzklopfen. Wer weiß, ob er einen nicht ansprechen wird? War das früher anders? Oder ist das erst unser Problem? Woher kommen diese Panzerungen?

Die Veröffentlichungen zur nationalsozialistischen Gesundheits-, Sozial- und Rassenpolitik - von Dörner, Roth, Wuttke-Groneberg, Klee und anderen - haben deutlich gemacht, daß es künftig in diesem Forschungsbereich vor allem auf Konkretion ankommen wird. Keine neuen Gesamt- und Überblicksdarstellungen sind gefragt, keine nochmalige Ausbreitung bekannter Materialien über diese »Medizin ohne Menschlichkeit", kein abermaliges Erschauern vor den MenschenVersuchern und "großen Tötern «; mit ihrer Aburteilung im Nürnberger ÄrzteProzeß hat die Offiziellen-Medizin, haben die Vertreter der Standeseliten in der deutschen Ärzteschaft ohnehin bereits das Thema als erledigt zu den Akten gelegt.

"Erledigt«? Worauf es jetzt ankommt, ist der Blick auf den Alltag der nazistischen "Volksgesundheits-Politik «: Die rationale Effizienz, ihr praktisches Funktionieren muß jetzt aus den Archivakten herausgekratzt werden - dieses unendlich verschachtelte bürokratische Getriebe mit all seinen kleinen und kleinsten Rädchen, die nach I945 weitergelaufen sind, als sei nichts geschehen, und die heute noch feiner, noch abgestimmter, noch effektiver zu arbeiten vermögen -, das muß ans Licht gezogen werden. Dazu sind zuerst konkrete Détail-Studien erforderlich, möglichst regional oder lokal begrenzt, um sich nicht wieder im allgemeinen zu verlieren.

Nach der Tübinger Projektgruppe ${ }^{I}$ und der großen Arbeit des Hamburger Vereins

\footnotetext{
* Brockkamp Verlag, Bremen 1984, 189 S., I9,80 DM.

I Volk und Gesundheıt. Heilen und Vernichten im Nationalsozialismus, hrsg. von der Projektgruppe "Volk und Gesundheıt«, Tübıngen 1982.
} 
zur Erforschung der NS-Sozialpolitik ${ }^{2}$ haben jetzt auch die beiden Bremer Ärzte Güse und Schmacke eine hervorragende Arbeit zur Geschichte der Zwangssterilisierung am Beispiel Bremens vorgelegt. Die Autoren, die schon vor Jahren zur Geschichte der Psychiatrie im Faschismus geschrieben hatten, stützen ihre Untersuchung vor allem auf Materialien des Bremer Staatsarchivs. Begleitet von den klangvollen und guten Wünschen des örtlichen Gesundheitssenators haben die Autoren die Bremer Archivalien durchgewühlt und bereits in den Generalakten so viel gefunden, daß es für ihre Arbeit mehr als genug gewesen ist. Freilich haben sie so manches nicht gesehen, nicht sehen können, weil die entsprechenden Akten auch für die wissenschaftliche Forschung - gesperrt sind. Man könnte meinen, dies sei zum Schutze der zwangssterilisierten Opfer geschehen - aber weit gefehlt: Der Persönlichkeitsschutz macht natürlich keinen Unterschied zwischen Opfern und Tätern. Wer die Akten des Erbgesundheitsgerıchts oder die Entnazifizierungsakten der verantwortlichen Ärzte und Juristen auswerten möchte, wird sich spätestens am Landesbeauftragten für den Datenschutz die Zähne ausbeißen.

"Gesetz zur Verhütung erbkranken Nachwuchses « - so heißt das Zwangssterilisierungsgesetz in der Nazi-Sprache. Wieviele Menschen aufgrund dieses Gesetzes unfruchtbar gemacht worden sind, ist nicht mehr genau zu ermitteln. 200000 bis $350000 \mathrm{Zwangssterilisierungen} \mathrm{sollen} \mathrm{es} \mathrm{im} \mathrm{gesamten} \mathrm{"Reichsgebiet} \mathrm{«} \mathrm{gewesen} \mathrm{sein.}$ Auch Bremen hat damals seinen Beitrag zur sogenannten "Aufartung" des deutschen Volkes geleistet. Die Bedingungen dafür waren nicht ungünstig. Trotz der heute gerne behaupteten hanseatischen Toleranz auf der einen und der Widerstandskraft gegenüber den Nationalsozialisten auf der anderen Seite waren in der Bremer Ärzteschaft genügend Rassenfanatiker, Herrenmenschen und Mit-Macher vertreten, um ein reibungsloses Funktionieren des Sterilisierungsgesetzes sicherzustellen. Ein zeitgenössischer Pressebericht über die erste Gautagung des NS-Ärztebundes mit feierlichem Senatsempfang im historischen Festsaal des Alten Rathauses vom Juni 1933 verdeutlicht das. Schon damals lauschten die im Braunhemd angetretenen Bremer NS-Ärzte den Tiraden über "Volksgesundheit « und "Vernichtung lebensunwerten Lebens « (S. 3 I/33).

Das »Gesetz zur Verhütung erbkranken Nachwuchses « vom 14.7.33 geht bekanntlich auf Gesetzesentwürfe und Diskussionen in der Weimarer Republik zurück. Dies hat es erleichtert, es nach dem "Zusammenbruch " nicht als "typisch nationalsozialistisches Unrecht « zu qualifizieren und auf diese Weise seine Opfer jahrzehntelang von jeder Entschädigungsregelung auszunehmen. Das Sterilisierungsgesetz selber enthält Formulierungen, die in ihrem Zynismus bemerkenswert sind. $\ 2$ : »Antragsberechtigt ist derjenige, der unfruchtbar gemacht werden soll.« - Wie soll man das nennen? Typisches Ordnungs- und Behörden-Deutsch, wenn es schon kein "typisch nationalsozialistisches Unrecht « sein soll? Natürlich ist von dieser Art von "Antragsrecht « praktisch kein Gebrauch gemacht worden; selbst dann, wenn die Betroffenen mit Dauereinweisung in die psychiatrischen Anstalten bedroht wurden, haben sie sich oft geweigert, einen "freiwilligen « Antrag auf Sterilisierung zu stellen. Dieser Anträge bedurfte es im Ergebnis natürlich auch nicht, denn $\ 3$ des Sterilisierungsgesetzes bestimmte: "Die Unfruchtbarmachung können auch beantragen: I. der beamtete Arzt, 2. für die Insassen einer Kranken-, Heil- oder Pflegeanstalt oder einer Strafanstalt der Anstaltsleiter.«

Diese Bestimmung bot nun die Rechtfertigungsgrundlage für die folgende Praxis von Denunziation und Heimlichkeit. Die Betroffenen haben in der weit überwie-

2 Angelika Ebbinghaus, Heidrun Kaupen-Haas, Karl Heinz Roth (hrsg.), Heilen und Vernuchten im Mustergau Hamburg. Bevölkerungs- und Gesundheıtspclitik im Dritten Reıch, Hamburg 1984. 
genden Mehrzahl der Fälle nie erfahren, von wem sie als »erbkrank« denunziert worden waren. Aus Einzelfällen ist ersichtlich, daß dort, wo die Betroffenen mit den Angaben der Denunzianten konfrontiert worden waren und sich dagegen zu wehren versuchten, sofort die NS-Behörden auf den Plan traten, um zu verhindern, daß die Anonymität der Antragsteller gelüftet werde. Als Antragsgründe nannte das Gesetz: Schwachsinn, Schizophrenie, manisch-depressives Irresein, Fallsucht, Veitstanz, angeborene Blind- oder Taubheit, körperliche Mißbildungen und Alkoholismus. Dieser Katalog ist in der Praxis weder vollständig gewesen, noch ist es wie Güse/Schmacke zeigen - in der Folgezeit hierbei geblieben. Neben der Unfruchtbarmachung wurde beispielsweise auch die Kastration bei Männern erlaubt, soweit der Betroffene von einem »entarteten Geschlechtstrieb befreit « werden sollte. Der Gesetzgeber nannte hierbei auch ausdrücklich Homosexualität als Indikation zur "freiwilligen Entmannung" (S. 37).

Was die NS-Ärzte unter »angeborenem Schwachsinn« diagnostisch untergebracht haben, illustriert die eigentliche Stoßrichtung des Sterilisierungsgesetzes: Aussonderung der "Leistungsschwachen « und »Randständigen «, der sozialen »Ballast-Existenzen «. "Mangelhafte Schul- und Berufsleistungen « oder sogenannte "Störungen der Gefühls- und Willens-Sphäre" genügten als diagnostische Generalklauseln bereits, Unangepaßte als »erbkrank « zu denunzieren. Die Heimlichkeit des Verfahrens, die die Denunzianten vor Unannehmlichkeiten mit den Betroffenen oder deren Angehörigen bewahrte, hat ein "antragsfreundliches Klima" geschaffen. Die Diagnose »angeborener Schwachsinn « stellte hierbei die am häufigsten angewandte Indikation dar. Auch "politisch Auffällige« wurden wegen »angeborenem Schwachsinn « denunziert - Begründung: »Schlechte Begriffs- und Urteilsbildung und abnorme Gefühls- und Willens-Sphäre" (S. 55 , I 52 ). Selbst Kinder sind von dem Gesetz nicht verschont geblieben; vom Io. Lebensjahr an konnten sie als »erbkrank « zwangsweise sterilisiert werden (S. $38 / 55$ ). Auch die Lehrer behinderter und lernschwacher Kinder scheinen hier ganze Arbeit geleistet zu haben. Nach den Ermittlungen von Güse/Schmacke wurden im Jahre 1934 im Raum Bremen allein $72 \%$ sämtlicher Hilfsschüler zwangssterilisiert (S.6I). Einzige Ausflucht für die von der Zwangsvorführung bedrohten Menschen war die freiwillige Anstaltsunterbringung - auf eigene Kosten versteht sich. In diesem Falle wurde das Verfahren vor dem Erbgesundheitsgericht für die Dauer der Anstaltsunterbringung unterbrochen. Die einer »Erbkrankheit" Geziehenen hatten also die Wahl, entweder der Unfruchtbarmachung zuzustimmen oder auf ihre persönliche Freiheit zu verzichten.

Was in all diesen Verfahren wirklich passiert ist, welche Rolle Eltern, Angehörige, Pfleger und Rechtsbeistände der Betroffenen spielten, ist bei der jetzigen Aktenlage praktisch nicht zu ermitteln. Solange die Akten der Erbgesundheitsgerichte nicht freigegeben werden, wird sich daran auch nichts ändern. Es wird daher in quantitativer Hinsicht schwer nachprüfbar sein, in wievielen der insgesamt 2677 Bremer Verfahren (1934-1944) die Unfruchtbarmachung als Druckmittel gegen sozial Unangepaßte mißbraucht worden ist. "Endogene (angeborene) Arbeitsscheu « wurde jedenfalls nach den Feststellungen von Güse/Schmacke als Form »verdeckten Schwachsinns" gedeutet (S. 77) und insofern als "Erbkrankheit« diagnostiziert. Es waren daher keineswegs nur die Leiter der Irrenanstalten, die ihre Patienten zur Zwangssterilisierung meldeten - auch die Sozialbehörden haben hierbei mitgewirkt (S. 80).

Unaufgeklärt und unaufklärbar ist der untergründige Zusammenhang zwischen Zwangssterilisierung und Euthanasie. Später, als die Bedingungen des »totalen Krieges « die letzten tödlichen Konsequenzen der Rassenhygiene ermöglichten, wird auch deutlicher, was mit den $Z$ wangssterilisierungen eigentlich beabsichtigt war. 
Friedemann Pfäfflin hat auf den Deportationslisten in die Tötungsanstalt von

Hadamar auch viele Namen von Patienten entdeckt, die einige Zeit zuvor von den Erbgesundheitsgerichten zur Zwangssterilisierung freigegeben worden waren.

Im Gegensatz zu den Ärzten und Richtern, die sich nach dem "Zusammenbruch" immer gerne auf den "regulären " Charakter des ja schon in der Weimarer Republik vorbereiteten "Erbgesundheitsgesetzes « berufen haben, wird den Betroffenen dieser dunkle Zusammenhang viel weitergehend bewußt gewesen sein, als dies von außen zu vermuten wäre. Die Fälle, in denen sich die zur Unfruchtbarmachung ausgewählten Frauen verzweifelt gegen den Eingriff zu wehren versuchten, scheinen nicht selten gewesen zu sein. Güse/Schmacke zitieren aus einer Krankenakte (S. I 2 I):

"Am I8. I. ist die W. R. aus Oberneuland ... zur Sterilisation hier aufgenommen worden. Da sie sehr unruhig war, wurde sie sofort auf die Beobachtungsstation verlegt und am 19. I. sterilisiert. Glatte einfache Operatıon. Wegen der starken motorischen Unruhe und des enormen Tobens und Schreiens mußte sie dauernd am Bett gefesselt werden und erhielt sie im Laufe des 20. I. I ccm Morph. Scolp... Am Morgen des 20. I. hatte die Patientin Fieber $40,2^{\circ}$, das von einer diffusen Bronchitis ... herrührte. Trotz laufender Verabreichung von Herzmitteln collabierte der Kreislauf mehrfach. Heute morgen stieg die Temperatur auf $42^{\circ}$. Unter sehr rascher Verschlechterung der Kresslaufverhältnisse trat um 7 Uhr der Exitus ein ...

Aufgrund der bei der Operation der Unfruchtbarzumachenden gemachten schlechten Erfahrungen halte ich es für richtig, wenn wir in Zukunft bei der z. Zt. herrschenden Grippe-Epidemie auf die Operation aller unruhigen und tobenden Frauen verzichten, die nur gefesselt und mit großen Gaben Narkotika im Bett gehalten werden können.*

Es ist nicht das einzige, in jener anheimelnden Mediziner-Sprache abgefaßte Dokument, das Güse/Schmacke ans Licht gezogen haben: "Patientin kam gestern früh ad exitum " - so endeten vor allem in Bremen überdurchschnittlich viele $Z$ wangssterilisierungen, weil einer der anfangs hauptbeteiligten Operateure durch Senilität und ein altersbedingtes Augenleiden besonders schwere Ausfälle zu verzeichnen hatte, ein Sachverhalt, der sogar die NS-Gesundheitsbürokratie zum Eingreifen veranlaßte (S. 128 ).

Die Geschichte der Zwangssterilisierungen endete nicht 1945. Güse/Schmacke schildern zunächst die Versuche, das Zwangs-Gesetz auch nach der Befreiung gegenüber der »verwahrlosten «, aus den Wirren der Nachkriegszeit hervorgekommenen Jugend weiterhin anzuwenden (S. 137). Auch damals gab es wieder - oder: noch - Ärzte, die die Unfruchtbarmachung von Randständigen befürworteten mit dem Argument, die »Leistungs- und Glücksfähigkeit« dieser Menschen sei beeinträchtigt (S. I43).

Güse/Schmacke behandeln sodann die Behörden-Odyssee der überlebenden Opfer, die sich um eine Entschädigung für das erlittene Unrecht bemüht hatten. Abgestempelt als "Renten-Neurotiker" hat man die Betroffenen bei den verschiedensten Ämtern herumgeschubst. Dort sind sie zuweilen wohl den gleichen Ärzten begegnet, die schon in Erbgesundheitsverfahren begutachtet hatten. Im deutschen Bundestag hat unterdessen der damalige Finanzminister Strauß begründet, warum auch eine Pauschalentschädigung nicht in Frage komme:

"Gegen eine Pauschalabfindung spricht überdies noch, daß von dem gesamten Entschädigungsbetrag von fast einer Milliarde DM bis zu $60 \%$ an Geisteskranke, Schwachsinnige oder schwere Alkoholiker gezahlt werden würde."

Mit der einige Zeit später dann doch bewilligten Pauschalentschädigung für die Zwangssterilisierten hat man sich wenigstens die Betroffenen vom Halse geschafft. Die in den Erbgesundheitsverfahren verantwortlichen Ärzte und Richter waren zu diesem Zeitpunkt freilich schon längst in den "wohlverdienten Ruhestand " getreten. 
Als ein Exempel für das, was nach dem "Zusammenbruch" von 1945 mit den Verantwortlichen geschehen ist, soll hier noch das sogenannte "Entnazifizierungsverfahren " des Vorsitzenden am Bremer Erbgesundheitsgericht Wilhelm Bolland geschildert werden. Dies ist sozusagen ein Nachtrag zu der Arbeit von Güse/ Schmacke, die die Entnazifizierungsakten nicht einsehen konnten, da sie für die Forschung mit dem erwähnten Argument des Persönlichkeitsschutzes gesperrt werden. ${ }^{3}$

Bolland, ein "Mann der ersten Stunde«, war nach sorgfältiger Durchleuchtung durch die Gestapo, die ihn offenbar für geeignet hielt, zum Vorsitzenden des Bremer Erbgesundheitsgerichts berufen worden. Er hat dieses Amt bis 1945 innegehabt. Unter seinem Vorsitz sind sämtliche Bremer Erbgesundheitsverfahren durchgeführt worden. Die Akte seiner »Entnazifizierung « beginnt mit einer persönlichen Eingabe an den Prüfungsausschuß für Beamtenentlassungen vom 21. I2. 1945; Bolland schreibt:

„Einspruch des Amtsgerichtsrats Wilhelm Bolland gegen die Anordnung der Militärregierung betr. Amtsentlassung, zugestellt am 28. November 1945 .

Gegen die oben bezeichnete Anordnung der Militärregierung, durch die mir die Ausübung des Richteramtes entzogen worden ist, lege ich hiermit Einspruch ein und bitte, mich zum Richteramt wieder zuzulassen ...

Nach meiner sicheren Überzeugung liegen die Voraussetzungen des Gesetzes Nr. 4 betr. Justizverwaltung in meinem Falle nicht vor.

Ich bin in die Partei eingetreten in der Auffassung, daß, wenn das Bürgerliche Element so stark wie möglich vertreten sei, nur so der Gefahr eines Radikalismus begegnet werden könne. Ich gestehe allerdings offen ein, daß ich geglaubt und gehofft habe, daß die nationalsozialistische Bewegung die ungesunden Klassengegensätze beseitigen und den sozialen Gedanken zum Wohle des Deutschen Volkes verwirklichen werde. Ich meine auch, daß meine politische Überzeugung hinreschend aus den mit dem letzten Fragebogen eingereichten Reden und Aufsätzen ... zu erkennen ist. (Anm.: Es handelt sich um Zeitungsartikel aus der Schlußphase der Weimarer Republik. Die Manuskripte seiner rassentheoretischen Vorträge hat Bolland nicht eingereicht) ... Ich habe der Parte1 im übrigen nur die regelmäßigen Beiträge gezahlt. Irgendwelche Förderung, Vorteile oder Vergünstigungen habe ich von der Partei weder erwartet noch erhalten.

An einer persönlichen Vernehmung, um die ich hiermıt bitte, wäre mir dringend gelegen, falls sie für erforderlich gehalten wird.

Über meine Persönlichkeit kann der mir von Jugend auf bekannte Tabakmakler ... Auskunft geben.«

Bolland ist trotz dieses Einspruchs nicht wieder in sein richterliches Amt zurückbefördert worden. Interniert wurde er allerdings auch nicht. So hatte er genug Zeit, sich auf das Entnazifizierungsverfahren zu konzentrieren. In einer Eingabe an die Bremer Spruchkammer vom 6.1.48 schreibt Bolland über seine Tätigkeit am Erbgesundheitsgericht:

*Ich habe die feste Überzeugung gehabt und habe sie jetzt noch, daß die Entscheidungen nach sorgfältiger medizinisch-wissenschaftlicher Erforschung des Sachverhalts gefällt wurden und daher zu verantworten waren, da ich meine Aufgabe, in jedem Falle die gesetzlichen Vorschriften des Verfahrens zu wahren, mit genau derselben peinlichen Gewissenhaftigkeit erfüllt habe, wie in allen anderen Fällen während meıner dreißigjährigen Tätigkeit als Richter. Ich bin bereit, mich auf das Zeugnis der gesamten Bremer Anwaltschaft dafür zu beziehen, daß diese meine Erklärungen Glauben verdienen. Ich weise auch an dieser Stelle jede widersprechende Vermutung oder gar Verdächtigung sehr entschieden zurück, umsomehr wenn sie von Laien ausgesprochen wird ... Im übrigen habe ich zu meinen sämtlichen Angaben ohne Ausnahme zu erklären, daß ich z. T. aus idealen, immer aber aus guten Motiven und anständigen Gründen gehandelt habe. Ich habe stets mein schweres Richteramt in Verantwortung vor Gott und meinem Gewissen ausgeübt. «

3 Die Akten, aus denen die folgenden Zitate stammen, liegen im Bremer Staatsarchiv unter 4,66 I. 
Auch der damalige Bremer Landgerichtspräsident Dr. Lahusen hat sich von Amts wegen zu der dienstlichen Tätigkeit Bollands geäußert. Er schrieb am 19.6.48 an die Spruchkammer:

"Mir Ist in meıner Amtstätigkeıt nichts davon bekannt geworden, daß der Amtsgerichtsrat Bolland sıch in seiner dienstlichen Tätıgkeıt, insbesondere bei der Urteilsfindung von anderen als unparteiischen und sachlichen Gesıchtspunkten hat leiten lassen. Es ist kein Fall bekannt, daß er etwa aus nationalsozialistıschen Gründen das Recht gebeugt oder eine unterschiedliche Behandlung der Rechtsuchenden hat eintreten lassen. Ich habe auch in meiner anwaltlichen Tätigkeıt niemals Anlaß gehabt, das Verhalten des Richters Bolland in irgendeiner Weise zu beanstanden.*

So kam es im Juni 1948 schließlich zu einer 3-tägigen Verhandlung vor der Bremer Spruchkammer. Als Rechtsbeistände hatte sich Bolland gleich zwei Verteidiger mitgebracht. Anders als in den Erbgesundheitsverfahren, in denen das Auftreten von Rechtsanwälten nur als »störend und verwirrend « kritisiert wurde (Güse/ Schmacke, S. 8r), hat es in den Entnazifizierungsverfahren solche Bedenken natürlich nicht mehr gegeben.

Als Zeugen wurden von der Spruchkammer vernommen: Der Leiter der Bremer Erziehungsanstalt (also ein Verfahrensbeteiligter, der selbst antragsberechtigt für Zwangssterilisierungen war), ein Richter, der mit Bolland das Dienstzimmer geteilt hatte ("... muß sagen, daß Richter Bolland gerechte Urteile herausgab und sich durch nichts beeinflussen ließ ... des Erbgesundheitsgerichts protokolliert hatten ( Die Termine wurden sehr menschlich durchgeführt, und die Kranken durften frei reden.«); ferner die Frau eines ehemaligen Untersuchungshäftlings und ein ehemaliger Untersuchungshäftling, die bekundeten, daß sie Ende 1944 von Bolland in seiner Eigenschaft als Untersuchungsrichter in Strafsachen vor dem Zugriff der Gestapo bewahrt worden seien; schließlich sind noch aufgetreten ein Bremer Hilfsschullehrer, der in den Erbgesundheitsverfahren als "Beistand " der Kranken auftreten sollte, und der Vater eines zwangssterilisierten Jungen. Damit ist die Reihe der Zeugen in dem Entnazifizierungsverfahren erschöpft; von den betroffenen Opfern ist kein einziges angehört worden.

Was in den Erbgesundheitsverfahren tatsächlich abgelaufen ist, läßt sich nach den Aussagen der beiden letzten Zeugen in Umrissen erahnen. Der als "Beistand " bestellte Hilfsschullehrer $\mathrm{K}$. hat ausgesagt:

"Zur Sache: Ich kenne den Richter Bolland von seiner Tätigkeit als Richter bei dem Erbgesundheitsgericht. Es war erwünscht, daß der Kranke durch einen Pfleger vertreten wurde. Aus meiner Einstellung heraus, habe ich das Amt als Pfleger angenommen. Ich habe an diesem Gerıcht beanstandet, daß dieses unter juristısche Leitung gestellt wurde, da die Termine dann nicht so menschlich durchgeführt werden konnten, wie es vielleicht für die Kranken sehr günstig gewesen wäre; denn diese Menschen sind sehr leicht durch Äußerlichkeiten zu beeinflussen. In einem Termin kam man als Pfleger nicht zum Zuge. Ich bin auch davon überzeugt, daß die ärztlichen Beisitzer zu entscheiden hatten, denn sie sind ja die Fachleute gewesen. Sicherlich lag der Verhandlung ein ärztliches Gutachten zugrunde. Der Pfleger hatte das Recht, Einsprüche zu erheben. Wenn ich vorher mit den Eltern gesprochen hatte, so war es nicht erforderlich, daß ich auf der Verhandlung anwesend war. Ich hatte ja sowieso auf der Verhandlung nıcht das Recht, mich zu Wort zu melden. Ich bin in meiner ersten Verhandlung, der ich beiwohnte, abgelehnt worden. Ich muß sagen, daß bei einer Verhandlung das Juristische überwogen hat. Sie wurde also nicht vertrauenserweckend geführt und dieses beeindruckte die Kranken. Mit dem Richter Bolland habe ich mich nicht dahingehend ausgesprochen, denn als Pfleger kam ich nicht mit ihm in Berührung. Ich bin vom Lehrerverein als Pfleger vorgeschlagen worden. In den meisten Fällen habe ich auf Rechtsmittel verzichtet, weil eine Berufung aussichtslos war. Messtens hatte ich vor der Verhandlung mit den Eltern gesprochen, die mit einer Sterilisierung einverstanden waren.* 
Nicht immer ist es den Rasse-Hygienikern gelungen, die Eltern zur Sterilisierung ihrer kranken Kinder zu bewegen. Dies zeigt die Aussage des am Schluß vernommenen Zeugen B:

"Zur Sache: Auf Grund einer Vorladung mußte mein I sjähriger Sohne 1936 zum Erbgesundhertsgericht kommen und dort wurde beschlossen, daß mein Sohn in eine Anstalt sollte, wo er vorher schon gewesen ist. Es ist möglich, daß eine Vorladung auf Veranlassung des Arztes aus Jena veranlaßt wurde. In Ellen fand der Termin unter Leitung des Richter Bolland, als Beisitzer Dr. Schomburg, statt. Ich habe vor Gericht ausgesagt, daß es sich um ein sehr schwächliches Kind handelt mit einer Zangengeburt. Eine erbliche Krankheit sei ausgeschlossen, da wir als Eltern gesund seien. Trotzdem lautete der Beschluß auf Unfruchtbarmachung wegen geisteskrank, mit dem ich absolut nıcht einverstanden war, da mein Kind sehr, sehr schwach war. Mir wurde erwidert, daß eine Sterilisierung auch in Amerika gemacht würde. Es wurde ein neuer Termin auf meine Beschwerde anberaumt, an dem menne Frau teilnahm. Die Entscheidung lautete, mein Sohn solle sofort in der Anstalt untergebracht werden, wenn ich mit der Sterilisierung nicht einverstanden war. Während einer Geschäftsreise ins Ausland hatte meine Frau eine Aufforderung erhalten, in der Löningstraße beim Amtsarzt zu erscheinen und dort wurde die Unfruchtbarmachung während meiner Abwesenheit durchgeführt. Nach der Unfruchtbarmachung bekam mein Kind Wechselfieber und ist gestorben. Ich bin der Ansicht, daß dieses auf die Unfruchtbarmachung zurückzuführen ist. Weiterhin bin ich mit Richter Bolland nicht zusammen gekommen.

Auf Vorbehalt des Beisitzers: Ich hatte seinerzeit den Eindruck, daß gleich bei Beginn der Verhandlung das Urteil schon fertig war. Eben gerade meine Abwesenheit, als mein Sohn unfruchtbar gemacht wurde, ist so schwerwiegend. Es ist doch erklärlich, daß wir uns nicht von unserem Kinde trennen wollten. Mein Sohn ist hier in Bremen nicht untersucht worden.«

Die Spruchkammer hat Bolland, der selber seine Einstufung als »Entlasteter « beantragt hatte, in die Gruppe der »Mitläufer « eingestuft, da er nur »formal belastet « sei; gleichzeitig wurde Bolland eine Geldsühne von 5o0,- DM, ersatzweise für je 20,-DM I Tag Sonderarbeit auferlegt. Die Höhe dieser Geldbuße entsprach in etwa einem halben Monatsgehalt, das der Verurteilte als Vorsitzender des Erbgesundheitsgerichts bezogen hatte. Dieser Spruch ist in Bremen auch durch die Presse gegangen. Der Weser-Kurier setzte am 26.6.48 im Anschluß an die Meldung eine Anmerkung der Redaktion:

*Als Beisitzer fungierte in dieser Verhandlung ein Mann, der bereits zweimal nach 1945 wegen Hühnerdiebstahls mit dem Strafrecht in Konflikt geraten war.*

Nach der Berufung des öffentlichen Anklägers ist die Entscheidung der Spruchkammer aufgehoben und durch die Berufungskammer folgendermaßen abgeändert worden:

"Der Betroffene ist Minderbelasteter gemäß Art. I I.

Es werden folgende Sühnemaßnahmen gegen ihn verhängt:

Es wird eine Bewährungsfrist von 2 Jahren festgesetzt. Während der Dauer der Bewährungsfrist ist dem Betroffenen untersagt:

a) ein Unternehmen als Inhaber, Gesellschafter, Vorstandsmitglied oder Geschäftsführer zu leiten oder ein Unternehmen zu beaufsichtigen oder zu kontrollieren, ein Unternehmen oder eine Beteiligung daran ganz oder teilweise zu erwerben;

b) in nicht selbständiger Stellung anders als in gewöhnlicher Arbeit beschäftigt zu sein;

c) als Lehrer, Prediger, Redakteur, Schriftsteller oder Rundfunk-Kommentator tätig zu sein.

3.) Es wird ein einmaliger Sonderbeitrag zu einem Wiedergutmachungsfond in Höhe von I000,-DM festgesetzt. Im Nichtbeitreibungsfalle tritt an die Stelle von je 5,- DM ein Tag Sonderarbeit für die Allgemeinheit.

4.) Der Streitwert beträgt auch für die Berufungsinstanz 8000,-DM.

5.) Der Betroffene hat auch die Kosten des Berufungsverfahrens zu tragen.*

In der Begründung ihres Spruches führt die Berufungskammer zunächst alle Mitgliedschaften Bollands in NS-Organisationen an und erklärt sodann: 
„Der Katalog der Mitgliedschaften zu soviel Organisationen des Naziregimes verbietet es nach Ansicht der Berufungskammer schon sprachlich, den Betroffenen als Mitläufer anzusehen. Durch diese Tatsache hat der Betroffene einwandfrei erwiesen, daß er dem Nationalsozialismus aus Überzeugung folgte und dessen Ideologie, Einrichtungen und Maßnahmen guthieß. Dies erhellt am klarsten der Umstand, daß der Betroffene sich von 1937-1945 als Mitarbeiter beim rassepolitischen Amt betätigte, einer Instıtution reın nazistischer Prägung. Für die Berufungskammer genügten diese Feststellungen für die Anwendbarkeit des Art. 7 I Ziff. 3 auf den Betroffenen, wobei bewußt von einer Beurteilung der Betätıgung des Betroffenen als Vorsitzender des Erbgesundheitsgerichts Abstand genommen wurde, da insoweit aufgetauchte Bedenken nucht eindeutig zu Ungunsten des Betroffenen auszulegen waren und somit nach dem Rechtsgrundsatz in dubio pro reo zu entscheiden gewesen wäre. Dem Betroffenen, der danach als Aktivist hätte eingestuft werden müssen, rechnete die Berufungskammer sein Verhalten in den Fällen ... und ... zugute, wenn hier auch zu unterstellen war, daß das Eintreten des Betroffenen für diese von der Gestapo Verfolgten zu einem Zeitpunkt erfolgte, als für den klar denkenden Beobachter die Hitlerherrschaft auf dem Aussterbeetat stand und jeder ,Gefolgsmann, danach trachten mußte, sich durch Guttaten gegenüber Andersdenkenden einigermaßen zu rehabilitieren. Insoweit griff die Berufungskammer auf die Erklärung des Landgerichtspräsidenten Dr. Lahusen (Bl. 4 d. A.) zurück, nach der der Betroffene sich in der letzten Zeit offensichtlich vom Nationalsozialismus abgewandt habe und wertete die Handlungsweise zu seinen Gunsten als eine Tat nach Art. 39 II Ziff. 4 des Gesetzes. Dieser besondere Umstand ließ den Betroffenen einer milderen Beurteilung würdig erscheinen und hatte seine Einstufung als Minderbelasteter gemäß Art. Ir zur Folge. Die Berufungskammer setzte eıne Bewährungsfrist von 2 Jahren fest, während derer dem Betroffenen Gelegenheit zum Nachweis seiner endgültigen Abkehr vom Nationalsozialismus und seiner Bereitschaft zur Erfüllung seiner Pflichten als Bürger ennes friedlichen demokratıschen Staates gegeben werden soll. In gerechter Abwägung der individuellen Verantwortlichkeit und der tatsächlichen Gesamthaltung wie auch unter Berücksichtigung des Alters und der wirtschaftlichen Verhältnisse des Betroffenen erschienen die nach Art. 17 verhängten Sühnemaßnahmen ausreichend und angemessen."

Der Spruch der Berufungskammer ist rechtskräftig geworden. Das heißt nun freilich nicht, daß die verhängten Sühnemaßnahmen auch realisiert worden wären. Im Nachverfahren wurde Bolland zunächst eine Ratenzahlung von 75,- DM pro Monat bewilligt; bei der Festsetzung der Höhe der Monatsraten wurde berücksichtigt, daß der Betroffene seit dem I.9.48 eine Abschlagszahlung auf seine zu erwartende Pension in Höhe von $250,-$ DM monatlich erhielt. Außerdem wurde die festgesetzte Bewährungsfrist von zwei Jahren auf drei Monate herabgesetzt.

Im Nachverfahren hatten sich allerdings auch noch einmal Betroffene von Erbgesundheitsverfahren gemeldet, um gegen die Entlastung Bollands zu protestieren. In einer Eingabe von Frau G. R. vom I6. I. 48 heißt es:

"Die Vernehmung meiner Tochter vor dem Erbgesundheitsgericht dauerte nur etwa 10 Minuten. Meine eigene Vernehmung ebenfalls nur dieselbe Zeit. «

Ein anderer Zwangssterilisierter, P.H., schrieb am II.4.49 über die Rolle seines "Beistands«, des Hilfsschullehrers K., dessen Zeugenaussage vor der Spruchkammer oben zitiert wurde:

"Er (der Pfleger K.) machte geltend, daß ich bei keınem Gericht gehört würde und daß jedes Beginnen in dieser Richtung zwecklos sei. Um der Sache noch die Krone aufzusetzen, sagte er mir noch, daß er verpflichtet se1, mich darauf aufmerksam zu machen, daß der NS-Staat die Sterilisierten als Märtyrer ansehen würde, weil sie für den Staat ein großes Opfer gebracht hätten. Dieses Opfer würde der Staat ihnen hoch anrechnen. Daß ein derartiges Opfer vom Musterstaat später mit Vergasung, Vergiftung und Einäscherung belohnt werden würde, konnte der dem System hörige Herr K. damals allerdings noch nıcht ahnen.*

Die Spruchkammer-Akte Bolland endet mit einem Gnadengesuch an den Bremer Bürgermeister Kaisen vom 5. Mai 1949, in dem der Verurteilte eine lebhafte Schilde- 
„Der Präsident des Senats

Es wird Ihnen mitgeteilt, daß der Herr Senatspräsident unter dem 6. dieses Monats im Gnadenwege die restlichen Sühne- und Kostenbeträge erlassen hat.*

Bolland hatte bis zu diesem Zeitpunkt von den Verfahrenskosten in Höhe von 992,-DM durch Ratenzahlungen insgesamt 325,-DM getilgt. Zahlungen auf die festgesetzte Sühne hatte er noch nicht geleistet. Sein "Entnazifizierungsverfahren« war damit abgeschlossen.

\title{
Reinhard Marx
}

\section{Eine menschenrechtliche Begründung des Asylrechts}

\author{
Rechtstheoretische und -dogmatische Untersuchungen zum \\ Politikbegriff im Asylrecht
}

Seit Jahren ist das politische Asyl eines der heftigst umstrittenen Themen der Innenpolitik. Die Abschirmung der Gesellschaft der Bundesrepublik ist vor dem Hintergrund der sich zuspitzenden internationalen Verteilungskrise zu sehen. Diese politischen Rahmenbedingungen des Asylrechts erzielen rechtlich über das Schlagwort "Ressourcenknappheit der Rechtsgewährung « praktische Wirkungen. Die Bundesrepublik bekennt sich jedoch zu den allgemeinen Menschenrechten. Politische Entscheidungen müssen daher menschenrechtlich gerechtfertigt werden. Das Asylrecht verdeutlicht aber mit aller Schärfe den Konflikt zwischen Politik und Menschenrechten: Im politischen Asyl geht es um das Politische schlechthin. Daher ist die Wirksamkeit dieses Grundrechts vom Politikverständnis der Gesellschaft abhängig. Das Asylrecht verdeutlicht zudem den politischen Mechanismus zwischen der Abschirmungs- und Expansionspolitik: Während die Nationalstaaten im wirtschaftlichen Bereich nach außen expandieren, schirmen sie sich in Fragen des internationalen Menschenrechtsschutzes ab.

Der Verfasser versucht, den Politikbegriff des Asylrechts vor diesem komplexen Hintergrund zu analysieren. Probleme ergeben sich vor allem daraus, daß mit den herkömmlichen Rechtsmethoden soziologische Hintergründe nur unzulänglich erfaßt werden können. Verschärfend kommt die auch in den Rechtswissenschaften bestehende starre Trennung zwischen innerer und äußerer Souveränität hinzu. Der Verfasser kommt zu dem Ergebnis, daß mit den traditionellen Rechtsmethoden der aslyrechtliche Politikbegriff nur unzureichend aufgearbeitet werden kann.

1984, 247 S., Salesta brosch., 49,-DM

ISBN 3-7890-0973-3

Nomos Verlagsgesellschaft

Postfach $610 \cdot 7570$ Baden-Baden

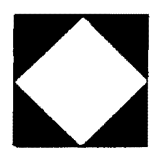

